\title{
RADIO STUDIES OF COSMIC RAYS IN THE GALAXY
}

\author{
W. REICH \\ Max-Planck-Institut für Radioastronomie \\ Auf dem Hügel 69 \\ D-5300 Bonn 1, FRG
}

\begin{abstract}
Changes of the cosmic ray electron spectrum throughout the Galaxy have been found, based on the comparison of large-scale radio continuum surveys. These observations are not compatible with the assumption of a static Galactic halo, but indicate the existence of a Galactic wind. Galactic plane surveys reveal sources of cosmic ray electrons in the Galactic disk. Recent studies of the population of radio sources show no evidence for a large number of compact Galactic non-thermal sources. Most of the extended sources are probably HII-regions. Relatively few new supernova remnants (SNRs) with low surface brightness could be identified. Most of the non-thermal emission in the disk-halo interface seems diffuse or unresolved, even at arcmin angular resolution.
\end{abstract}

\section{INTRODUCTION}

The radio continuum emission observed from the Galaxy for frequencies up to a few $\mathrm{GHz}$ is dominated by the synchrotron emission process. Thermal emission is seen within a few degrees of the Galactic plane. The intensity of synchrotron emission depends on the number of cosmic ray electrons and the magnetic field component perpendicular to the line of sight. It decreases with frequency for a power law electron energy spectrum $\mathrm{N} \sim \mathrm{E}^{-\gamma}$, with a direct relation of the energy spectral index $y$ to the observed brightness temperature spectral index $\beta=(1-y) / 2+2$. $\beta$ is calculated from the measured brightness temperatures $\mathrm{T}_{\nu} \sim \nu^{-\beta}$ at two frequencies $v$.

Information on the radio emission from the Galaxy is based on two types of surveys. All-sky or large-scale surveys at medium angular resolutions have been made to study the disk and the high latitude emission, but also show large local structures like the giant radio loops. Because of our position inside in the Galaxy these surveys need absolute calibration. Model fits are required to get an idea of the three-dimensional distribution of the Galactic emissivity. A comparison of surveys at different frequencies results in spectral indices of the various Galactic components.

Sources for cosmic ray electrons are supposed to be highly concentrated in the Galactic plane. To resolve the mixture of diffuse emission and the large concentration of discrete emission complexes in the Galactic 
plane higher angular resolution surveys are needed. Galactic plane surveys are the basis for the identification of sources.

\section{LARGE SCALE GALACTIC RADIO CONTINUUM SURVEYS}

A list of absolutely calibrated large-scale surveys is given in Table 1. At present the all-sky survey at $408 \mathrm{MHz}$ has the highest angular resolution and sensitivity. The $1420 \mathrm{MHz}$ survey of the northern sky has a somewhat higher angular resolution and matches the $408 \mathrm{MHz}$ survey in sensitivity. Observational problems increase with frequency and the listed $2.3 \mathrm{GHz}$ and $2.7 \mathrm{GHz}$ surveys are likely to be the limit for ground-based sensitive large-scale continuum surveys.

The $408 \mathrm{MHz}$ survey has been used by Phillipps et al. (1981) and Beuermann et al. (1985) to obtain three-dimensional models of the Galactic emissivity. Their results are somewhat different, especially in respect to the $\mathrm{z}$-extent and strength of the thick disk or halo component of the Galaxy. Thick disks or halos around galaxies are certainly better studied on the basis of sensitive radio observations of edge-on galaxies, which have become available during the last years (Hummel, this volume).

TABLE 1 .

Large-Scale Surveys (HPBW $<11^{\circ}$ )

\begin{tabular}{|c|c|c|c|c|}
\hline $\begin{array}{c}\text { Frequency } \\
{[\mathrm{MHz}]}\end{array}$ & $\begin{array}{l}\text { HPBW } \\
{\left[0^{\circ}\right]}\end{array}$ & $\begin{array}{c}\text { Sensitivity } \\
{[\mathrm{K}]}\end{array}$ & $\begin{array}{c}\text { Declination } \\
\text { Coverage }\end{array}$ & Authors \\
\hline 10 & $2.6 \times 1.9 \mathrm{secz}$ & 20000 & $-5^{\circ}$ to $74^{\circ} *$ & Caswell 1976 \\
\hline 30 & 11 & 1000 & all-sky & Cane 1978 \\
\hline 38 & $7.25 \times 8.25$ & 400 & $>-25^{\circ}$ & Milogradov-Turin et al. 73,84 \\
\hline 85 & $3.8 \times 3.5$ & 100 & all-sky & Yates 1968 \\
\hline 150 & 2.2 & 25 & all-sky & Landecker, Wielebinski 1970 \\
\hline 178 & $0.22 \times 4.6$ & 20 & $-5^{\circ}$ to $88^{\circ}$ & Turtle, Baldwin 1962 \\
\hline 404 & $8.5 \times 6.5$ & 3 & $>-20^{\circ}$ & Pauliny-Toth, Shakeshaft 1962 \\
\hline 408 & 0.85 & 2 & all-sky & Haslam et al. 1982 \\
\hline 820 & 1.2 & 0.5 & $-7^{\circ}$ to $85^{\circ}$ & Berkhuijsen 1972 \\
\hline 1420 & 0.59 & 0.05 & $>-19^{\circ}$ & Reich 1982, Reich, Reich 1986 \\
\hline 2300 & 0.33 & 0.04 & southern sky* & Jonas et al. 1985 \\
\hline 2700 & 0.3 & 0.03 & $\begin{array}{l}\text { northern sky* } \\
\text { *incomplete }\end{array}$ & Reif et al. 1987 \\
\hline
\end{tabular}

References: Berkhuijsen 1972, Astr. Astrophys. Suppl. 5, 263; Cane 1978, Aust. J. Phys. 31, 561; Caswell 1976, Mon. Not. R. Astr. Soc. 177, 601; Haslam et al. 1982, Astr. Astrophys. Suppl. 47, 1; Jonas et al. 1985, Astr. Astrophys. Suppl. 62, 105; Landecker, Wielebinski 1970, Aust. J. Phys. Astr. Suppl. 16, 1; Milogradov-Turin, Smith 1973, Mon. Not. R. Astr. Soc. 161, 269; Milogradov-Turin 1984, Mon. Not. R. Astr. Soc. 208, 379; Pauliny-Toth, Shakeshaft 1962, Mon. Not. R. Astr. Soc. 124, 61; Reich, W. 1982, Astr. Astrophys. Suppl. 48, 219; Reich, P., Reich, W. 1986, Astr. Astrophys. Suppl. 63, 205; Reif et al. 1987, Mitt. Astr. Ges. 70, 419; Turtle, Baldwin 1962, Mon. Not. R. Astr. Soc. 124, 36; Yates 1968, Aust. J. Phys. 21, 167 


\section{SPECTRAL INDEX VARIATIONS IN THE GALAXY}

Variations of the radio spectral index with Galactic latitude have been interpreted in terms of a disk-halo model of the Galaxy (Webster, 1975; Sironi, 1976; Strong, 1977; Webster, 1978). These investigations are mainly based on low resolution drift scans at low frequencies. Since the derived spectra indicate some steepening with Galactic latitude, the data have been interpreted in terms of a flat spectrum disk and a steep spectrum halo component. However, the quality of the data used seems poor if compared with more recent observations (Lawson et al., 1987; Reich and Reich, 1988a,b) and in addition the spectra suffer from confusion of the Galactic background emission with emission from the giant radio loops. In fact, it has been shown in a spectral analysis by Milogradov-Turin (1985, 1987) that a steep spectrum halo is not in agreement with the data, if the influence of the steep spectrum North Polar Spur is taken into account. Lawson et al. (1987) presented a newly calculated spectral index map between $38 \mathrm{MHz}$ and $408 \mathrm{MHz}$, which shows slightly steeper spectra at higher latitudes than in the Galactic plane $(\Delta \beta=0.1)$ mostly in the regions of the giant radio loops. Globally, flatter Galactic spectra are observed at low frequencies rather than at higher frequencies. This reflects the steepening of the electron energy spectrum with increasing energy (Webber, 1983). Even if the electron spectrum at high $z$ is the same as in the Galactic plane, steeper radio spectra at high $\mathrm{z}$ are expected. It is evident from the characteristic frequency $v_{c} \sim B \mathrm{E}^{2}$ that a decrease of the magnetic field strength $B$ with $z$ requires radiating electrons with higher energies $\mathrm{E}$ than in the Galactic plane.

The most detailed map of Galactic spectral indices across the northern sky has been calculated by Reich and Reich (1988a) based on the 408 $\mathrm{MHz}$ and the $1420 \mathrm{MHz}$ surveys. Figure 1 shows the $1420 \mathrm{MHz}$ data and the distribution of temperature spectral indices. The map of spectral indices shows significant variations along the Galactic plane, and from the plane towards higher Galactic latitudes. It has been discussed in detail by Reich and Reich (1988b). Besides some local structures steep spectra are seen in the first Galactic quadrant $\left(1<55^{\circ},|b|<20^{\circ}\right)$. Correcting for thermal and foreground emission a spectral index of $\beta=3.1$ was found for the non-thermal emission in the inner part of the Galactic. disk. This result is in close agreement with that of Hirabayashi (1974), who found $\beta=3.08 \pm 0.11$ for the non-thermal spectrum between $1.4 \mathrm{GHz}$ and $10 \mathrm{GHz}$. The non-thermal emission in the second and third quadrant has a spectral index close to $\beta=2.85$ in the plane and the spectra flatten continously with increasing Galactic latitude. The flattest spectra are seen at $1 \sim 230^{\circ}, b \sim 30^{\circ}$ and $1 \sim 200^{\circ}, b \sim-45^{\circ}$. As concluded by Reich and Reich $(1988 \mathrm{~b})$, these results are unexpected for a static or purely convective Galactic halo, but agree to some extent with the cooling-convection halo models as proposed by Lerche and Schlickeiser (1982). These models are based on the existence of a Galactic wind.

It is of interest that a spectral analysis by Bloemen et al. (1988) of the $y$-ray emssion as observed by the COS-B satellite, shows a similar spectral flattening with increasing $\mathrm{z}$ in the second and third quadrant as found for the radio data. This suggests a hardening of both the cosmic ray proton and electron spectrum with increasing $\mathrm{z}$ at $\mathrm{GeV}$ energies in 
1420 MHZ SURVEY AT AN ANGULAR RESOLUTION OF $2: 5$

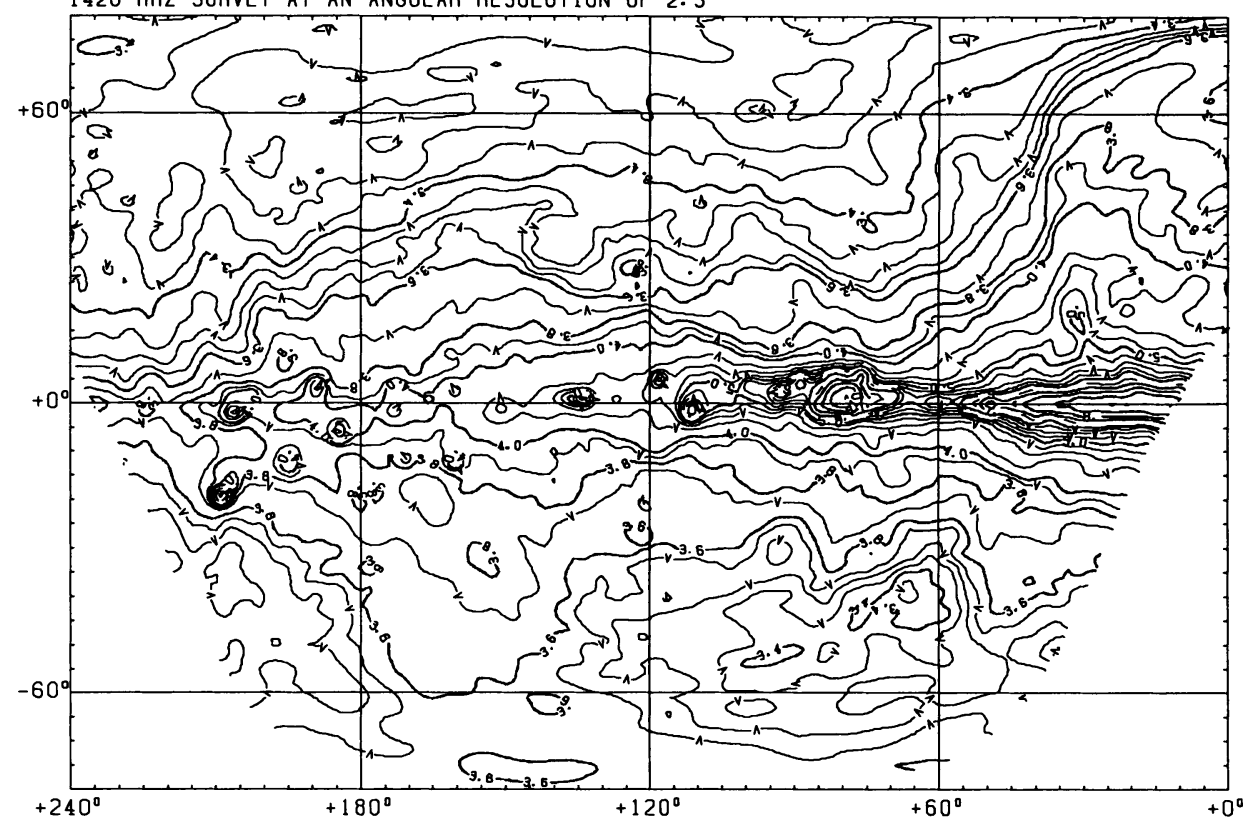

SPECTRAL INDEX MAP AT A RESOLUtion OF 2:5 408/1420 MHZ

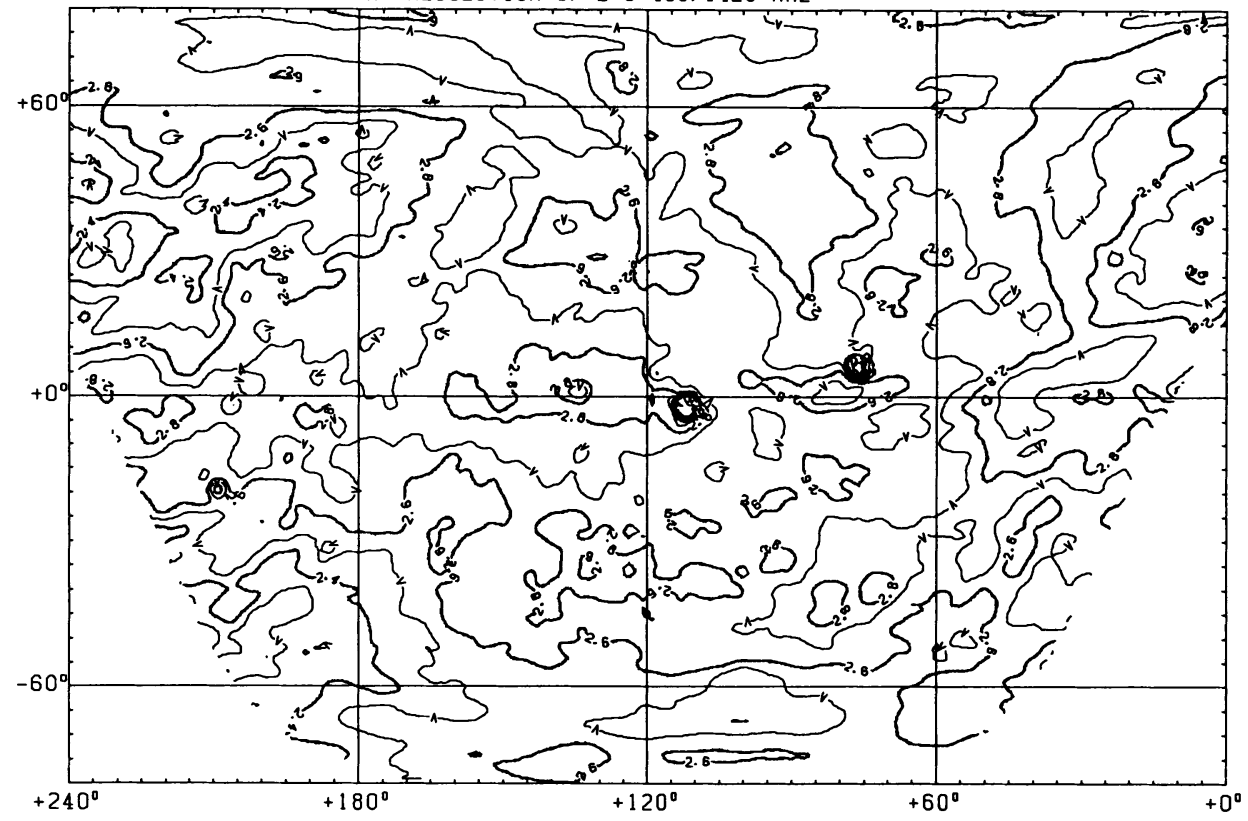

Figure 1. Coordinates are Galactic. Contours are in $K T_{B}$ (top) and represent temperature spectral indices (bottom) (see Reich and Reich, 1988a,b). 
the outer Galaxy, which further supports the asumption of the existence of a Galactic wind.

Reich and Reich (1988b) have used the edge-on view of the Galaxy as modelled by Beuermann et al. (1985) from the $408 \mathrm{MHz}$ survey and decomposed the brightness temperatures along the plane into an inner and outer non-thermal component with spectral indices of $\beta=3.1$ and $\beta=$ 2.85 , and a thermal component for the inner disk. Figure 2 shows the predicted spectral index variations. The rather small spectral differences result from the superposition of the three components in the line of sight. A very wide frequency coverage is necessary to sort out the three components even for our simple model. It is obvious that Galactic wind effects in edge-on galaxies must be much more pronounced than in our Galaxy if they are to be observable.

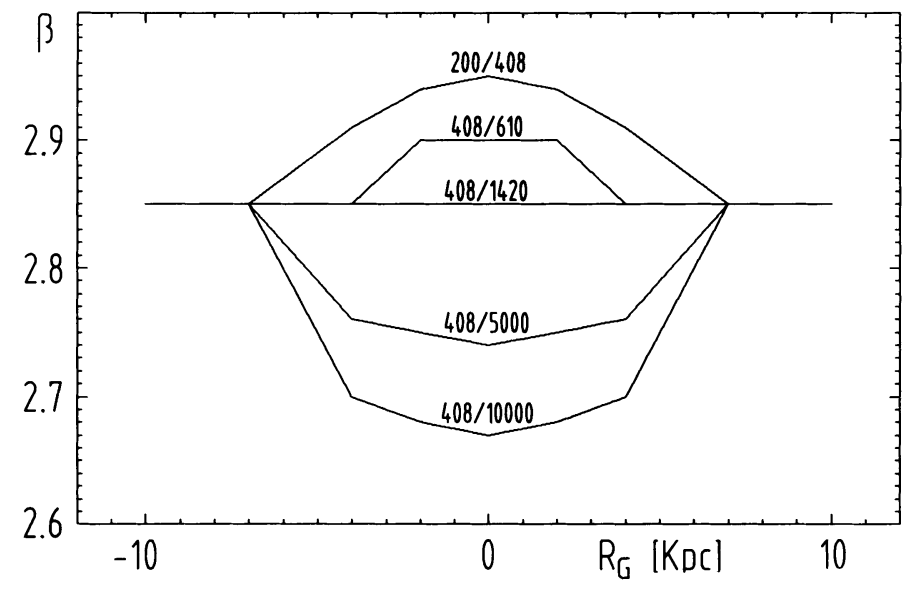

Figure 2. Variation of the temperature spectral index $\beta$ of the Galactic disk emission when seen edge-on. The frequency pairs are indicated.

\section{GALACTIC PLANE SURVEYS}

Galactic plane surveys with arcminute resolution are listed in Table 2 . Highest angular resolution has been obtained at 4.9 and $10 \mathrm{GHz}$, which is required to see details within complex structures. Even higher resolution data come from synthesis telescopes, where some source surveys are currently in progress. The maps with highest sensitivity are from the Effelsberg $1.4 \mathrm{GHz}$ and $2.7 \mathrm{GHz}$ surveys. A large number of sources has been listed. The identification of these sources is a long- term project, but. global statistical results can be given.

\section{COMPONENTS OF THE GALACTIC DISK EMISSON}

Figure 3 shows the number distribution of compact sources with a peak flux density larger than $40 \mathrm{mJy}$ at $2.7 \mathrm{GHz}$ as a function of Galactic latitude. For $1<100^{\circ}$ there is an excess of about 900 sources at low latitudes with intrinsic sizes between $2: 5$ to $11^{\prime}$. The scale height is about $0^{\circ} .6$. A weak excess is seen for sources with sizes between $1: 3$ and $2: 5$ for latitudes below $0: 5$. Unresolved sources show no excess, although 
TABLE 2 .

Selected Single Dish Galactic Plane Surveys (HPBW ( $10^{\prime}$ )

\begin{tabular}{|c|c|c|c|c|}
\hline $\begin{array}{l}\text { Frequency } \\
{[\quad[\mathrm{GHz}]}\end{array}$ & $\begin{array}{l}\text { HPBW } \\
{[']}\end{array}$ & $\begin{array}{l}\text { Sensitivity } \\
{[\mathrm{K}]}\end{array}$ & 1 Coverage & Authors / Telescope \\
\hline 1.4 & 9 & 0.2 & $\begin{array}{c}93^{\circ}-162^{\circ} \quad 4^{\circ} \\
236 \text { sources }\end{array}$ & $\begin{array}{l}\text { Kallas, Reich } 1980 \\
\text { Effelsberg } 100-\mathrm{m}\end{array}$ \\
\hline 1.4 & 9.4 & 0.125 & $\begin{array}{c}357^{\circ}-95.5^{\circ} 4^{\circ} \\
884 \text { sources }\end{array}$ & $\begin{array}{l}\text { Reich et al 1990a } \\
\text { Effelsberg } 100-\mathrm{m}\end{array}$ \\
\hline 2.7 & 8.2 & 0.2 & $\begin{array}{c}190^{\circ}-61^{\circ} 2^{\circ} \\
890 \text { sources }\end{array}$ & $\begin{array}{l}\text { various see Day et al } 1972 \\
\text { Parkes } 64-\mathrm{m}\end{array}$ \\
\hline 2.7 & 4.3 & 0.05 & $\begin{array}{c}357.4^{\circ}-240^{\circ} 5^{\circ} \\
6483 \text { sources }\end{array}$ & $\begin{array}{l}\text { Reich et al } 1984,1990 \mathrm{~b} \\
\text { Fürst et al 1990a,b } \\
\text { Effelsberg } 100-\mathrm{m}\end{array}$ \\
\hline 4.9 & 2.6 & 0.2 & $\begin{array}{r}357.5^{\circ}-60^{\circ} 1^{\circ} \\
1186 \text { sources }\end{array}$ & $\begin{array}{l}\text { Altenhoff et al } 1978 \\
\text { Effelsberg } 100-\mathrm{m}\end{array}$ \\
\hline 5 & 4.1 & 0.2 & $\begin{array}{c}190^{\circ}-40^{\circ} 2^{\circ} \\
915 \text { sources }\end{array}$ & $\begin{array}{l}\text { Haynes et al } 1978 \\
\text { Parkes } 64-\mathrm{m}\end{array}$ \\
\hline 10 & 2.7 & 0.1 & $\begin{array}{c}356^{\circ}-56^{\circ} 1.5^{\circ} \\
144 \text { sources }^{\circ}\end{array}$ & $\begin{array}{c}\text { Handa et al } 1987 \\
\text { Nobeyama } 45-\mathrm{m}\end{array}$ \\
\hline
\end{tabular}

References: Altenhoff et al. 1978, Astr. Astrophys. Suppl. 35, 23; Day et al. 1972, Austr. J. Phys. Astr. Suppl. 25, 1; Fürst et al., 1990a,b Astr. Astrophys. Suppl. in press (October vol.); Handa et al. 1987, Publ. Astr. Soc. Japan 39, 709; Haynes et al. 1978, Austr. J. Phys. Astr. Suppl. 45, 1; Kallas, Reich 1980, Astr. Astrophys. Suppl. 42, 227; Reich et al. 1984, Astr. Astrophys. Suppl. 58, 197; Reich et al. 1990a, Astr. Astrophys. Suppl. 83, 539; Reich et al. 1990b, Astr. Astrophys. Suppl. in press (October vol.)

some weaker sources are likely to be missed in some highly confused areas of the Galactic plane. Garwood et al. (1988) have used the VLA to make a source survey at $1.5 \mathrm{GHz}$. They found an excess of about $20 \%$ of sources for $1<40^{\circ}$ above the extragalactic source counts. This excess is due to compact structures towards identified thermal sources. Figure 3 shows for $l>100^{\circ}$ a quite uniform source distribution with latitude. Source counts for small diameter sources with sizes less than 2:5 agree within a few percent with extragalactic source counts (Fürst et al., 1990). High resolution VLA observations of 135 sources smaller than 2 ' in the anticentre direction by Fich (1986) resulted in the identification of 11 HII-regions, but no compact SNR. These statistical results indicate a paucity of compact non-thermal Galactic sources, which might be young far distant SNRs, or more exotic objects like SS433 or Sco X-1.

The Effelsberg surveys have been used to identify extended low surface brightness SNRs. Confusion is lowest in the anticentre direction and SNRs with a surface brightness as low as $\Sigma_{1 \mathrm{GHz}} \sim 2.10^{-22} \mathrm{~W} \mathrm{~m}^{-2}$ $\mathrm{Hz}^{-1} \mathrm{sr}^{-1}$ are clearly visible, but only three sources are seen within $90^{\circ} \leqslant 1 \leqslant 240^{\circ}$. The surface brightness of the Galactic background emission is more than ten times higher and the few observable low surface brightness SNRs imply a minor contribution of this class of sources to the unresolved background. A number of new SNRs could be identified in the 

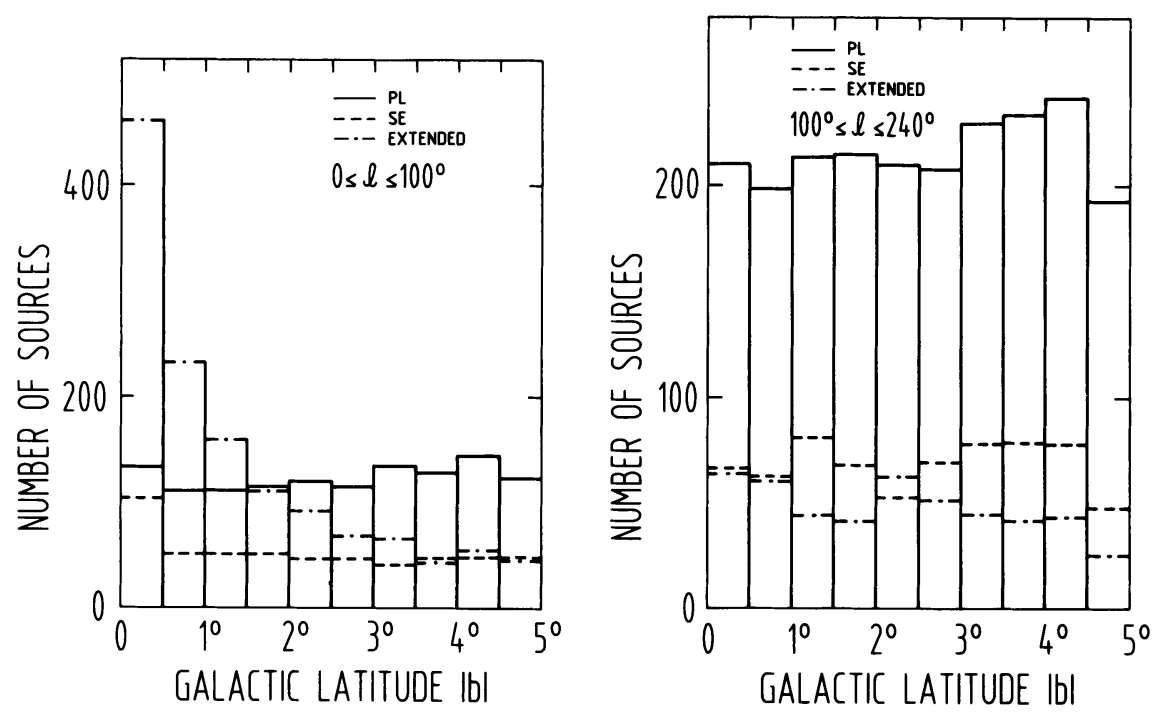

Figure 3. The number of radio sources at $2.7 \mathrm{GHz}$ versus absolute Galactic latitude. Point-like (PL) sources are unresolved with intrinsic sizes less than 1.3. Slightly extended (SE) sources have intrinsic sizes between $1: 3$ and 2.5 . Extended sources have sizes between 2.5 and $11^{\prime}$.

first galactic quadrant (Reich et al., 1988). Omitting the highly confused regions with $|b| \leqslant 0.5$ the distribution of $\Sigma$ for "known" sources (Green, 1988) and "new" ones is given in Table 3.

TABLE 3.

Distribution of SNR Surface Brightness

$357^{\circ}<1<76^{\circ}, 0: 5 \leqslant|b| \leqslant 5^{\circ}$

\begin{tabular}{lcccc}
\hline$-\log (\Sigma)$ & $<19$ & $19-20$ & $20-21$ & $21-21.7$ \\
"known" & 1 & 5 & 8 & 3 \\
"new" & 1 & 1 & 13 & 13 \\
all SNRs & 2 & 6 & 21 & 16 \\
\hline
\end{tabular}

The "new" SNRs are much weaker, on average, than those previously detected. No distances are known for most of the sources. Diameters may be obtained by applying a $\Sigma-D$ relation for shell-type sources. Using the relation given by Milne (1979) cumulative counts $\mathrm{N}(<D)$ can be made. For adiabatic expansion $\mathrm{N}\left(\langle\mathrm{D}) \sim \mathrm{D}^{5 / 2}\right.$ is expected, which is seen for diameters up to $40 \mathrm{pc}$. Based on the $\Sigma-D$ relation only about five sources from Table 3 have larger diameters than $40 \mathrm{pc}$. Again there is no indication for the existence of numerous faint large diameter SNRs, which contribute significantly to the non-thermal disk emission. 
Figure 4 shows the latitude dependence of the integrated $1.4 \mathrm{GHz}$, $2.7 \mathrm{GHz}$ and IRAS $60 \mu \mathrm{m}$ emission for $4^{\circ} \leqslant l \leqslant 36^{\circ}$. Data are at 9.4 angular resolution and have been scaled relative to $1.4 \mathrm{GHz}$ for optically thin thermal emission. A flux density ratio of $\mathrm{S}_{60} \mu \mathrm{m} / \mathrm{S}_{2.7} \mathrm{GHz}=1000$, which is typical for thermal emission in this longitude range, has been assumed (Fürst et al., 1987). Also shown is the integrated source emission obtained after subtraction of the smooth large-scale emission. About half of the total emission at $b=0^{\circ}$ is from discrete sources. Their thermal fraction is close to $90 \%$. HII-regions are the major source contri-
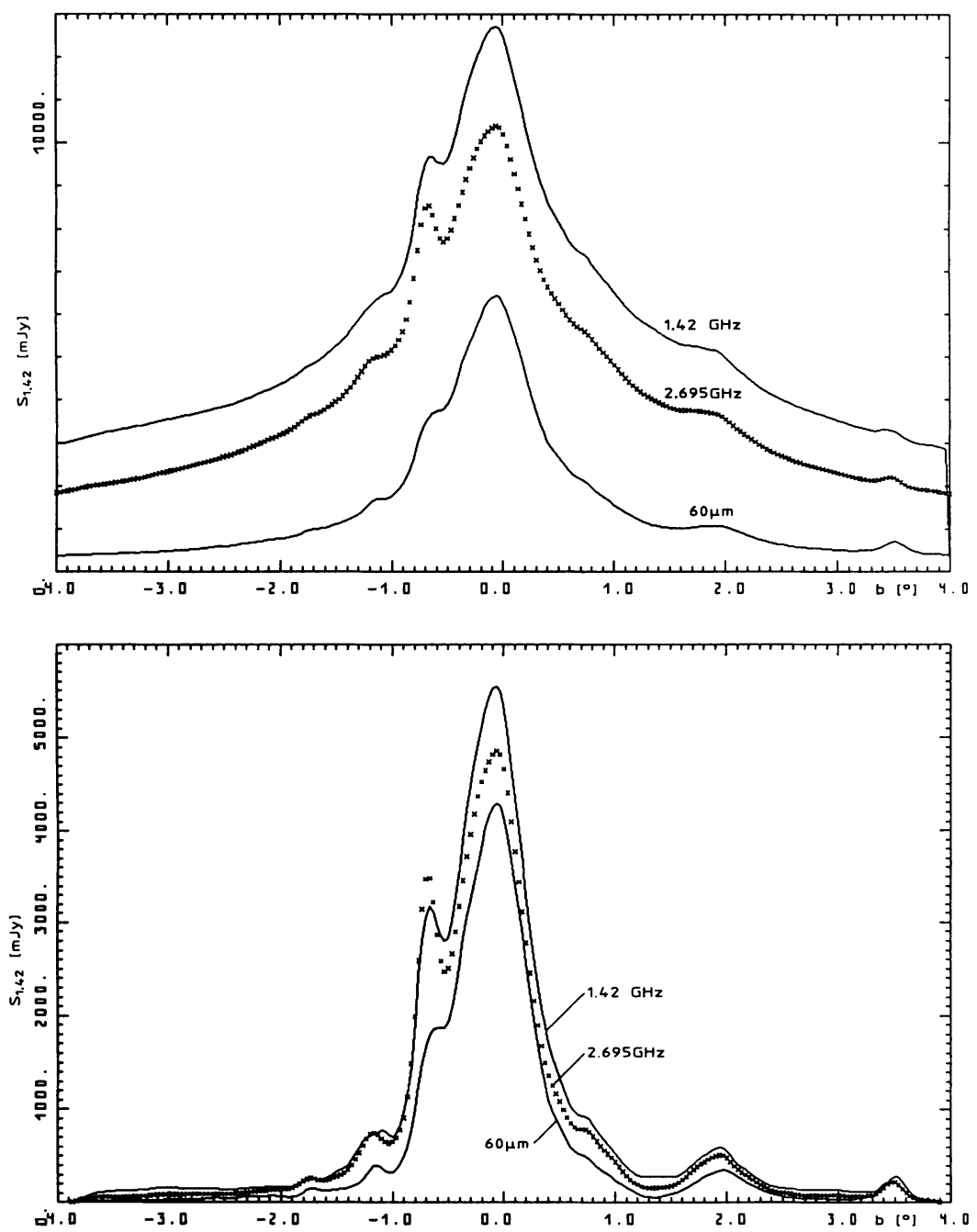

Figure 4. Top: Integrated Galactic emission (see text). $\left(\mathrm{T}_{\mathrm{B}} / \mathrm{S}\right)_{1.4} \mathrm{GHz}=2$. Bottom: Integrated emission from discrete sources. 
bution in this frequency range. The discrete source component contributes only a few percent to the total emission for $|b|>1^{\circ}$.

In Figure 5 the $1.4 \mathrm{GHz}$ and $2.7 \mathrm{GHz}$ data at $b=-2: 5$ are shown, with separate plots for the small-scale and the extended emission. The data are scaled for thermal emission. The non-thermal nature of most of the small-scale and the diffuse emission is evident. The survey maps show that the small-scale emission at a few degrees of Galactic latitude
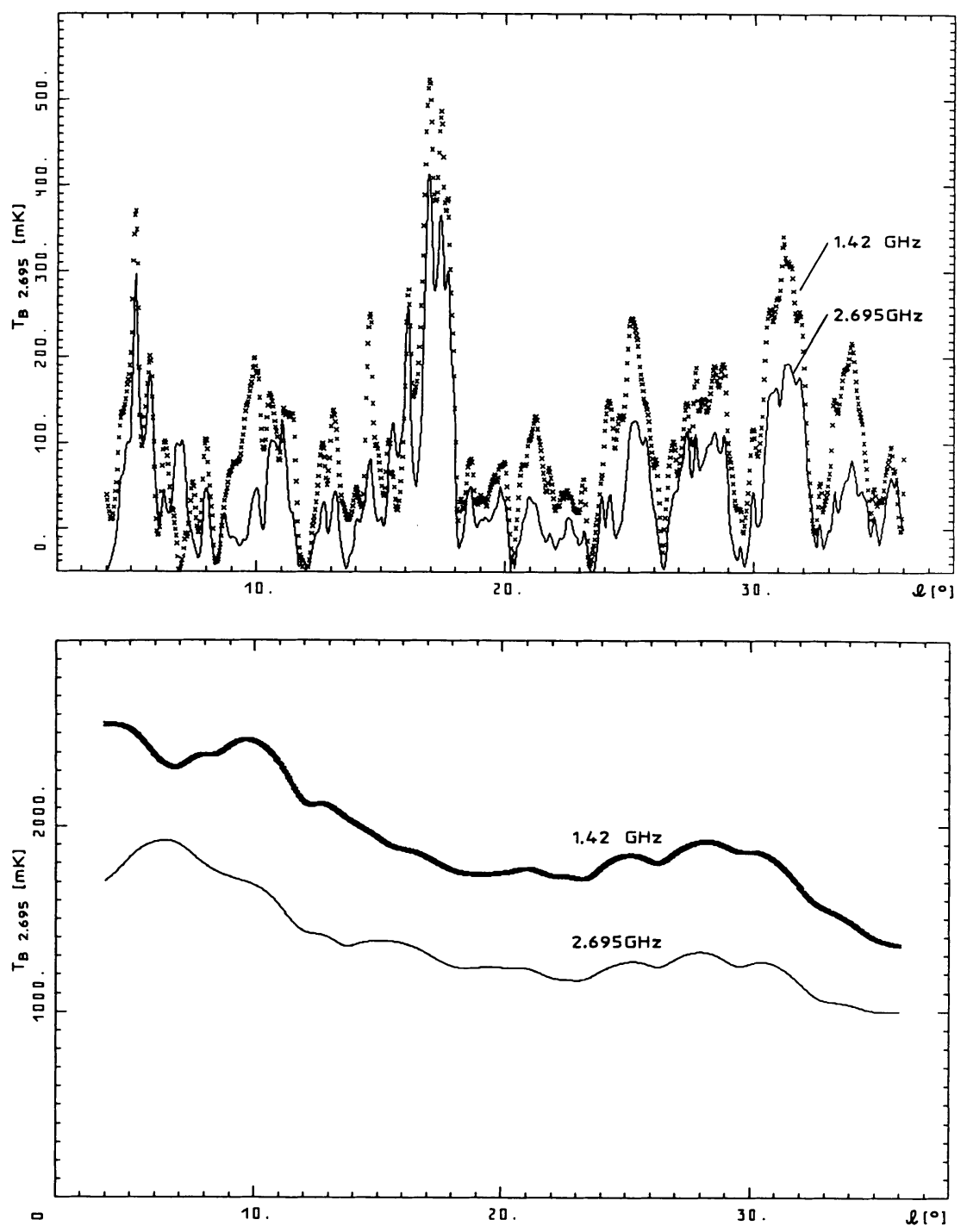

Figure 5. Small-scale (top) and large-scale emission (bottom) at $b=-2: 5$. The $1.4 \mathrm{GHz}$ data have been scaled relative to $2.7 \mathrm{GHz}$ for optically thin thermal emission. 
is rather complex and can not be classified in a simple scheme. Its average fraction is of the order of $5 \%$ of the diffuse emission.

To summarize: The observed synchrotron emission a few degrees outside of the Galactic plane remains basically unresolved, even when observed with high sensitivity at arcmin angular resolution. This indicates rather small fluctuations in the magnetic field strength and the cosmic ray electron density in the disk-halo interface. A mainly uniform outflow of cosmic ray electrons from the disk sources into the halo is consistent with the observations. Alternatively, a convection of cosmic ray electrons from clustered supernova explosions in $\mathrm{OB}$-associations via chimneys into the halo has been recently proposed (e.g. Norman and Ikeuchi, 1989). In view of the smooth radio emission these chimneys have to be sufficiently numerous and/or large in size with small internal fluctuations in the magnetic field strength and electron distribution to agree with the observations.

\section{REFERENCES}

Beuermann, K., Kanbach, G., Berkhuijsen, E.M. (1985) Astron. Astrophys. 77,25

Bloemen, J.B.G.M., Reich, P., Reich, W., Schlickeiser, R. (1988) Astron. Astrophys. 204, 88

Fich, M. (1986) Astron. J. 92, 787

Fürst, E., Reich, W., Sofue, Y. (1987) Astron. Astrophys. Suppl. 71, 63

Fürst, E., Reich, W., Reich, P., Reif, K. (1990) Astron. Astrophys. Suppl., in press (October volume)

Garwood, R.W., Perley, R.A., Dickey, J.M., Murray, M.A. (1988) Astron. J. 96,1655

Green, D.A. (1988) Astrophys. Space Sci. 148, 3

Hirabayashi, H. (1974) Publ. Astron. Soc. Japan, 26, 263

Lawson, K.D., Mayer, C.J., Osborne, J.L., Parkinson, M.L. (1987) Monthly Not. Roy. Astron. Soc. 225, 307

Lerche, I., Schlickeiser, R. (1982) Astron. Astrophys. 107, 148

Milne, D. (1979) Australian I. Phys. 32, 83

Milogradov-Turin, J. (1985) in Proc. IAU Symposium No. 106, eds. H. van Woerden, R.J. Allen, W.B. Burton, Reidel, Dordrecht, p. 245

Milogradov-Turin, J. (1987) in Proc. of the 10th ERAM, ed. J. Palous, Czechoslovak Acad. Sc., Praha, p. 225

Norman, C.A., Ikeuchi, S. (1989) Astrophys. J. 345, 372

Phillipps, S., Kearsey, S., Osborne, J.L., Haslam, C.G.T., Stoffel, H. (1981) Astron. Astrophys. 103, 405

Reich, P., Reich, W. (1988a) Astron. Astrophys. Suppl. 74, 7

Reich, P., Reich, W. (1988b) Astron. Astrophys. 196, 211

Reich, W., Fürst, E., Peich, P., Junkes, N. (1988) in Proc. IAU Coll. No. 101, eds. R.S. Roger, T.L. Landecker, Cambridge Univ. Press, p. 293

Sironi, G. (1976) Astrophys. Space Sci. 44, 159

Strong, A. (1977) Monthly Not. Roy. Astron. Soc. 181, 311

Webber, W.R. (1983) in Composition and Origin of Cosmic Rays, ed. M.M.

Shapiro, Reidel, Dordrecht, p. 83

Webster, A. (1975) Monthly Not. Roy. Astron. Soc. 171, 243

Webster, A. (1978) Monthly Not. Roy. Astron. Soc. 185, 507 